\title{
Assessment of Identity During Adolescence Using Daily Diary Methods: Measurement Invariance Across Time and Sex
}

\author{
Andrik I. Becht, Susan J. T. Branje, \\ and Wilma A. M. Vollebergh \\ Utrecht University
}

\author{
Jaap J. A. Denissen \\ Tilburg University
}

\author{
Dominique F. Maciejewski, Pol A. C. van Lier, \\ and Hans M. Koot \\ VU University Amsterdam
}

Wim H. J. Meeus

Utrecht University and Tilburg University

\begin{abstract}
The aim of this study was to assess measurement invariance of adolescents' daily reports on identity across time and sex. Adolescents $(N=497$; mean age $=13.32$ years at Time $1,56.7 \%$ boys $)$ from the general population reported on their identity commitments, exploration in depth and reconsideration on a daily basis for 3 weeks within 1 year across 5 years. We used the single-item version of the Utrecht Management of Identity Commitments Scale (UMICS; Klimstra et al., 2010), a broad measure of identity-formation processes covering both interpersonal and educational identity domains. This study tested configural, metric, scalar, and strict measurement invariance across days within weeks, across sex, across weeks within years, and across years. Results indicated that daily diary reports show strict measurement invariance across days, across weeks within years, across years, and across boys and girls. These results support the use of daily diary methods to assess identity at various time intervals ranging from days to years and across sex. Results are discussed with regard to future implications to study identity processes, both on smaller and larger time intervals.
\end{abstract}

Keywords: measurement invariance, daily diary reports, identity, adolescence

One of the key tasks of adolescents is to develop a mature identity (Erikson, 1968). Initiated by several factors, one of which is cognitive development, adolescents increasingly explore their identities (Lichtwarck-Aschoff, van Geert, Bosma, \& Kunnen, 2008). Studies investigating the development of identity across adolescence have mainly focused on long-term development of identity formation using relatively large time intervals (e.g., Kroger, Martinussen, \& Marcia,

This article was published Online First August 10, 2015.

Andrik I. Becht and Susan J. T. Branje, Research Centre Adolescent Development, Utrecht University; Wilma A. M. Vollebergh, Department of Interdisciplinary Social Sciences, Utrecht University; Dominique F. Maciejewski, Pol A. C. van Lier, and Hans M. Koot, Department of Developmental Psychology, EMGO Institute for Health and Care Research, VU University Amsterdam; Jaap J. A. Denissen, Department of Developmental Psychology, Tilburg University; Wim H. J. Meeus, Research Centre Adolescent Development, Utrecht University and Department of Developmental Psychology, Tilburg University.

Data of the RADAR study were used. RADAR has been financially supported by main grants from the Netherlands Organisation for Scientific Research (GB-MAGW 480-03-005), and Stichting Achmea Slachtoffer en Samenleving (SASS) to RADAR PIs, and from the Netherlands Organisation for Scientific Research to the Consortium Individual Development (CID; 024.001.003).

Correspondence concerning this article should be addressed to Andrik I. Becht, Research Centre Adolescent Development, Utrecht University, PO Box 80 140, 3508 TC Utrecht, the Netherlands. E-mail: a.i.becht@uu.nl
2010; Meeus, van de Schoot, Keijsers, Schwartz, \& Branje, 2010). However, an important principle of life-span developmental science is that short-term variability is a driving force of longer term developmental change (Nesselroade \& Molenaar, 2010). Echoing this, identity researchers acknowledge the importance of studying both shortterm dynamics and long-term development of identity (LichtwarckAschoff et al., 2008). Focusing on this short-term development of identity, some studies have started using daily diary methods to measure identity in adolescence (e.g., Klimstra et al., 2010; Yip \& Fuligni, 2002), which can also be used to study short-term dynamics across longer periods (e.g., across years). Moreover, with regard to clinical relevance, identity has become an explicit core maker in the description of personality disorders in the Diagnostic and Statistical Manual of Mental Disorders (5th ed.; DSM-5; American Psychiatric Association, 2013; Feenstra, Hutsebaut, Verheul, \& van Limbeek, 2014). For example, patients with borderline personality disorder have been characterized by identity disturbances, such as inconsistencies in their thoughts, feelings, and behaviors (e.g., Vater, SchröderAbé, Weißgerber, Roepke, \& Schütz, 2015; Wilkinson-Ryan, \& Westen, 2000). Therefore, it might be of clinical relevance to investigate whether short-term identity fluctuations can be used to identify early signals of problematic personality development. Similarly, dayto-day variability in adolescents' identity predicts higher levels of anxiety and depression over time (Schwartz et al., 2011). In addition, daily reports may be used to further investigate gender differences in the patterns of identity formation (Meeus et al., 2010) within shorter time intervals. 
However, to make valid comparisons on identity scores over time or across sex, it is important to assess whether these daily diary assessments measure the same construct (i.e., identity) over time and across boys and girls. Specifically, to be able to make such comparisons, such daily diary questionnaires must show measurement invariance, implying that they measure an identical construct with the same structure across time and/or groups (van de Schoot, Lugtig, \& Hox, 2012). For instance, items such as pushing and biting might be indicators of physical aggression at age 4 , whereas at age 12 , these items will no longer adequately reflect the construct of physical aggression. Developmental change in constructs may also occur within smaller time intervals (e.g., weeks, years), especially in adolescence, an important developmental period involving transitions in the peer system, the family system, and school (Graber, Brooks-Gunn, \& Petersen, 1996). Applying the investigation of measurement invariance to the construct of identity, it is possible that, when adolescents mature, certain items of the daily diary identity measure acquire a different meaning and consequently a different score. For instance, questions about certainty with regard to friendships may be more important in younger adolescents given the importance of peers in identity development during early adolescence than in older adolescents. In other words, items that capture adolescents' identity may change across adolescence. Similarly, differences may exist between boys and girls in the interpretation of the items that measure identity.

Therefore, the aim of the present study was to investigate shortand long-term longitudinal invariance and invariance across sex of daily diary assessments of identity across adolescence.

\section{The Need to Study Short-Term Development of Identity}

Previous research investigating the development of identity across adolescence has mainly focused on long-term processes of identity formation, in which relatively large time intervals were used (e.g., Meeus et al., 2010). Recently, however, identity researchers have stressed the theoretical relevance to study identity processes at shorter time intervals to uncover potentially interesting variance in identity development both within and between adolescents (Lichtwarck-Aschoff et al., 2008). For example, according to the Meeus-Crocetti model (Crocetti, Rubini, \& Meeus, 2008), identity formation can be seen as a dynamic between certainty and uncertainty, operating on a daily basis (LichtwarckAschoff et al., 2008). From day to day, adolescents explore and weigh various possible commitments against one another, and decide whether commitments need to be changed (Luyckx, Goossens, \& Soenens, 2006; Meeus et al., 2010). Furthermore, shortterm assessments of identity can be used to model identity development across longer time intervals across adolescence.

Focusing on short-term processes, day-to-day reports on identity may be aggregated into short-term variability scores or aggregated mean scores (e.g., per week). In line with the use of aggregated scores, research on conflict frequency in adolescents shows that assessments with short time intervals (e.g., daily, weekly) provide the most reliable assessment strategy (Burk, Denissen, Van Doorn, Branje, \& Laursen, 2009). Applying the use of daily diary reports to identity, short time-interval assessments may more directly adhere to dynamics of identity for- mation than annual interval assessments. For example, it has been suggested that young adults move back and forth between different identity statuses within short time intervals. These movements are also referred to as moratorium-achievementmoratorium-achievement cycles (MAMA-cycles; Stephen, Fraser, \& Marcia, 1992). These MAMA-cycles are expected to operate especially within short time intervals before and around specific life events, for example, when adolescents experience difficulties with friends or at school. Whereas annual assessments of identity might not uncover important variability in adolescents' identity formation, identity assessments with shorter time intervals might be better able to tap into this cyclic nature of the process of identity development.

In addition to studying mean-level processes of identity formation, daily diary methods that assess day-to-day variability in identity formation may be necessary to study its links with adolescents' adjustment outcomes. Campbell (1990) for example, suggested that mean levels of adolescent self-concept are less important than the variability of self-concept. In line with this suggestion for self-concept, the mean level of identity commitments might be a less important predictor of adolescents' adjustment than short-term variability of commitments because large fluctuations in identity-formation processes may impair adolescents' adjustments in daily life. In line with theoretical models that acknowledge the importance to study short-term processes of identity formation, several studies have started using daily diary assessments of identity. For example, studies that were based on the same data set as the current study, found that more day-to-day fluctuations in identity reconsideration predicted higher mean levels of reconsideration and lower mean levels of commitment across years (Klimstra et al., 2010). Moreover, day-to-day fluctuations in reconsideration and commitment predict higher levels of anxiety and depression over time (Schwartz et al., 2011). These studies showed that short-term identity dynamics are important in influencing identity-formation and adjustment outcomes of adolescents in the long run.

Although microlevel approaches are increasingly used to study short-term processes within identity development, it has not yet been investigated whether these daily dairy methods can actually be used to make valid comparisons on identity processes across short time intervals (e.g., weeks). Moreover, these daily reports can also be used to study short-term dynamics across longer periods (e.g., across years). For example, daily reports could be used to compare early adolescents with late adolescents in identityformation processes. However, if measurement invariance was not established, differences in mean levels of identity across time may be due to measurement artifacts rather than true change over time.

In addition to investigating longitudinal invariance of daily reports, it is unclear whether possible differences between boys and girls can be validly assessed with daily diary methods. For example, using annual reports on identity, girls are often found to be further ahead in their identity formation process than boys (Meeus et al., 2010). Daily reports may further inform us about specific underlying processes that might explain differences in identity between boys and girls. However, if measurement invariance across sex is not established, we cannot draw certain conclusions about differences between boys and girls, which might result from differences in the meaning of the assessed construct (i.e., identity), rather than true differences between boys and girls. To 
make sure that the construct of identity is being measured in the same way across different time points and across sex, one has to investigate whether these daily diary assessments of identity show measurement invariance. To the best of our knowledge, the current study is the first to investigate longitudinal invariance and invariance across sex of daily diary assessments of identity across adolescence (ages 13-18)

\section{The Present Study}

The current study contributes to the literature on the use of daily diary reports on identity by investigating measurement invariance across time and sex. Four research questions were addressed to explore these issues. First, we examined whether daily diary reports on adolescent's identity showed measurement invariance across days within weeks. Second, we examined whether daily reports showed measurement invariance across days within weeks for boys and girls. Third, we analyzed measurement invariance of daily reports across weeks within years. Fourth, we examined whether daily reports of identity showed measurement invariance across 5 years.

In this study, we adopted the three-dimensional Meeus-Crocetti model of identity formation (Crocetti et al., 2008). Commitment refers to strong choices that adolescents have made with respect to various developmental domains. Exploration in depth refers to the extent to which adolescents reflect on the commitments they already have made by actively exploring those commitments. Reconsideration refers to the willingness to discard current commitments in favor of searching for new ones. We investigated measurement invariance of daily identity reports using broad measures of identity covering two important developmental domains (i.e., interpersonal and educational domains) of identity formation within adolescence. The interpersonal domain taps into commitments, exploration in depth, and reconsideration with regard to the relationship with a best friend. The interpersonal domain was introduced into identity research in the 1980s by Grotevant (1987). The second domain is about educational identity, dealing with commitments, exploration in depth, and reconsideration with regard to adolescents' education.

Because this study used daily reports across adolescence, we were able to investigate both measurement invariance of daily reports at short time intervals (e.g., across weeks) and across larger time intervals (e.g., across years).

\section{Method}

\section{Participants and Procedure}

Participants were 497 Dutch adolescents (282 boys, 56.7\%), with a mean age of 13.32 years $(S D=0.46$, range $11.29-15.84$ years) at the first measurement year, who enrolled in the young cohort of the ongoing longitudinal project for Research on Adolescent Development and Relationships-Young Cohort (RADARY). Adolescents were recruited from central and western parts of the Netherlands. Most adolescents came from medium to high socioeconomic-status (SES) families $(89.2 \%)$ and had a Dutch background $(94.8 \%)$. The present study used data from ages $13-$ 18. Over the course of 5 years, adolescents participated in 15 Internet-based measurement weeks (3 weeks of Internet assess- ment within each of the 5 years). Each measurement week, participants filled out a questionnaire tapping into identity for 5 days in a row (i.e., Monday through Friday) resulting in 75 Internetbased assessments in total. The first Internet-assessment week took place in June, 2006; the second and third Internet-assessment weeks took place 3 and 6 months later, respectively. This same measurement interval for filling out the questionnaires was used across the 5 years. To complete the online assessments, participants had to $\log$ on to a website where they could fill out the Internet assessment. During the Internet-assessment weeks adolescents were reminded of participation by sending an e-mail invitation around 5:30 p.m. If adolescents did not complete the assessment $1.5 \mathrm{hr}$ after the first e-mail invitation, they received an additional reminder e-mail. If adolescents still had not completed the Internet assessment, they received text messages and phone calls after an additional $1.5 \mathrm{hr}$. Adolescents received $€ 10$ (equivalent to US \$11) for participation per assessment week. All participants agreed to participate by signing an informed consent form. Participants were informed that they could discontinue participation at any time.

Concerning sample attrition, $19.6 \%$ of the adolescents that participated in the 1 st measurement year dropped out in the 5 th measurement year. No significant differences were found with regard to distribution of boys and girls, $\chi^{2}(1)=0.51, p=.476$, between adolescents who participated in the 1 st year but not in the 5 th year, compared with adolescents who were still participating in the 5th year. However, adolescents who dropped out over the course of the study were more likely to come from lower SES families, $\chi^{2}(1)=12.76, p<.001$. Furthermore, a multivariate analysis of variance (MANOVA) was computed with the mean identity scales of the first assessment week (i.e., for commitment, exploration in depth and reconsideration in both interpersonal and educational domains) and age as dependent variables, which showed a significant result, $F(7,449)=3.177 ; p=.003$. Subsequent post hoc tests revealed that adolescents who dropped out over the course of the study were slightly older (i.e., 0.22 years), $F(1,457)=17.122, p<.001$, compared with adolescents who were still participating in the 5 th year. No significant differences on any of the identity scales were found (all $p \mathrm{~s}>.09$ ).

\section{Measures}

Daily identity assessment. To measure identity on a daily basis, the single-item version of the Utrecht Management of Identity Commitments Scale was used (U-MICS; Klimstra et al., 2010). Adolescents reported on a 5-point Likert scale ( $1=$ completely untrue, 5 = completely true) using one item per identity dimension (i.e., commitment, exploration in depth, and reconsideration) for both the interpersonal (i.e., relation with best friend) and educational (i.e., school) domains (six items in total per day). The item for commitment was, "Today, I felt confident about myself because of my best friend/education" (interpersonal/educational commitment); for exploration in depth, "Today, I often thought about my best friend/education" (interpersonal/educational exploration in depth), and reconsideration: "Today, I felt that I could better look for a different best friend/education" (interpersonal/ educational reconsideration).

We used the procedure developed by Heise (1969) to compute reliabilities of single-item scales. In this procedure, the reliability 
of a single-item scale is assessed with an estimate of the test-retest reliability using the following formula: $r_{\mathrm{xx}}=\left(r_{12} \times r_{23}\right) / r_{13}$, which separates true change from measurement error. We calculated the Heise reliabilities for the 1st year. Because there were three measurement weeks with 5 days each, we computed three Heise estimates per identity domain per week. With three measurement weeks within one year, this resulted in nine estimates per identity domain. For the single-item subscales of commitment, exploration in depth and reconsideration in the 1st year, the mean Heise reliabilities ranged between .68 and .85 for interpersonal identity and between .63 and .74 for educational identity. These Heise reliabilities are in line with previous reports on identity (Klimstra et al., 2010; Schwartz et al., 2011).

\section{Missing Data}

Missing data points per day occurred for $31.5 \%$ of participant assessments across the study. Due to the substantial number of data points relative to the number of observations, Little's missing completely at random (MCAR) test was conducted on the study variables per year. The pattern of missing data values was completely random, with the normed $\chi^{2}\left(\chi^{2} / d f\right)$ ranging between 1.02 and 1.05 per year, indicating a good fit between the imputed and nonimputed sample scores (Bollen, 1989). Because we found support for MCAR in our sample, we chose to use all available data points per participant by including all of them in the analyses. Full-information likelihood (FIML) was used for our main analyses.

\section{Statistical Analyses}

Because we assessed each identity dimension with a single item, we were able to investigate whether these questions, repeatedly assessed from Monday through Friday, were equally important indicators of a weekly latent identity factor.

Longitudinal invariance testing. To test whether adolescent's daily reports on identity scales showed measurement invariance over time, a hierarchical set of models was fit with increasingly stringent equality constraints (Vandenberg \& Lance, 2000). In the first baseline model, configural invariance is assessed to determine whether the same factors and pattern of factor loadings exist over time (Little, 2013). With configural invariance, there are no equality constraints imposed on parameters. Configural invariance is typically established if the specified configuration of the model shows acceptable fit to the data. The second model tests for metric invariance by examining whether the magnitude of the factor loadings is equal over time. If metric invariance is established, the implication is that the items are equally important contributors to the factor total scores over time, which makes it possible to compare relationships between total scores. The third model tests for scalar invariance by additionally constraining the indicator intercepts to be equal across time. When scalar invariance holds, the implication is that differences on the items are only due to differences on the common factors. Therefore, when scalar invariance is assessed, the mean scores of daily identity assessments can be compared between different time points. In the fourth model, the strict invariance test is performed, which constrains the residual variances to be equal across time in addition to the factor loadings and intercepts. When strict invariance is established, differences between time points can be measured with the same amount of error (van de Schoot et al., 2012).

\section{Invariance Across Boys and Girls}

To establish whether daily reports on identity are measurementinvariant across sex, another hierarchical set of models was fitted, with each subsequent step imposing additional constraints to the model. For the first baseline model, configural invariance was assessed to test whether the same three-factor structure of identity existed for boys and girls. In the second model, metric invariance was tested to establish whether the magnitude of the factor loadings was equal for boys and girls. In the third model, scalar invariance was investigated to test whether the indicator intercepts were equal for boys and girls. In the fourth model, scalar invariance was examined, in which the residual variances were constrained to be equal for boys and girls.

Invariance over time and/or across sex was considered to be present when adding the constraints did not result in a significant decrease of model fit. First, initial model fit of the configural model was determined with three goodness-of-fit indices: The comparative fit index (CFI), the root mean-square error of estimation (RMSEA), and the standardized root mean-square residual (SRMR; Cheung \& Rensvold, 2002). Fit indices were deemed adequate using the following cutoffs: CFI $>.90$, RMSEA $<.08$, and SRMR $<.08$ (Byrne, 2012). Second, we assessed changes in fit indices to determine invariance at each subsequent stage of testing: $\Delta \mathrm{CFI} \geq-.01, \Delta \mathrm{RMSEA} \geq .015$, and $\Delta$ SRMR $\geq .030$ (Chen, 2007; Cheung \& Rensvold, 2002; Little, 2013). If two or more change scores were below the specified threshold, invariance was assumed. We used the robust MLR estimator to account for non-normal distributions (Muthén \& Muthén, 1998-2012). Given our large sample, and because the Satorra-Bentler $\chi^{2}\left(\mathrm{~S}-\mathrm{B} \chi^{2}\right)$ statistic is sensitive to sample size, we only used the $\mathrm{S}-\mathrm{B} \chi^{2}$ and the $\mathrm{S}-\mathrm{B} \chi^{2}$ difference test $\left(\Delta \mathrm{S}-\mathrm{B} \chi^{2}\right)$ for descriptive purposes (Chen, 2007; Cheung \& Rensvold, 2002; Kline, 2010).

Measurement models. Four different measurement models were used to assess measurement invariance of daily ratings on identity across (a) 5 days within 15 separate measurement weeks, (b) 5 days within 15 separate measurement weeks for boys and girls, (c) 3 measurement weeks within 5 years, and (d) 5 years using confirmatory factor analysis (CFA) in Mplus 7 (Muthén \& Muthén, 1998-2012). These models were tested for interpersonal and educational identity separately.

In the first set of models, longitudinal measurement invariance was tested across days within 15 separate measurement weeks for both interpersonal and educational identity. In this model, the five daily indicator items, all covering 1 day of the week (i.e., Monday through Friday) were forced to load on the associated weekly identity factor (i.e., commitment, exploration in depth, or reconsideration). This factor structure was the same for every measurement week (see Figure 1).

In the second set of models, we tested for measurement invariance of interpersonal (15 models, one model per measurement week) and educational identity (15 models, one model per measurement week) of days within weeks for boys and girls. Figure 2 represents the estimated multigroup model, showing that per week the same factor model was specified for boys and girls. Table 2 displays the results for every step of testing for measurement invariance across boys and 


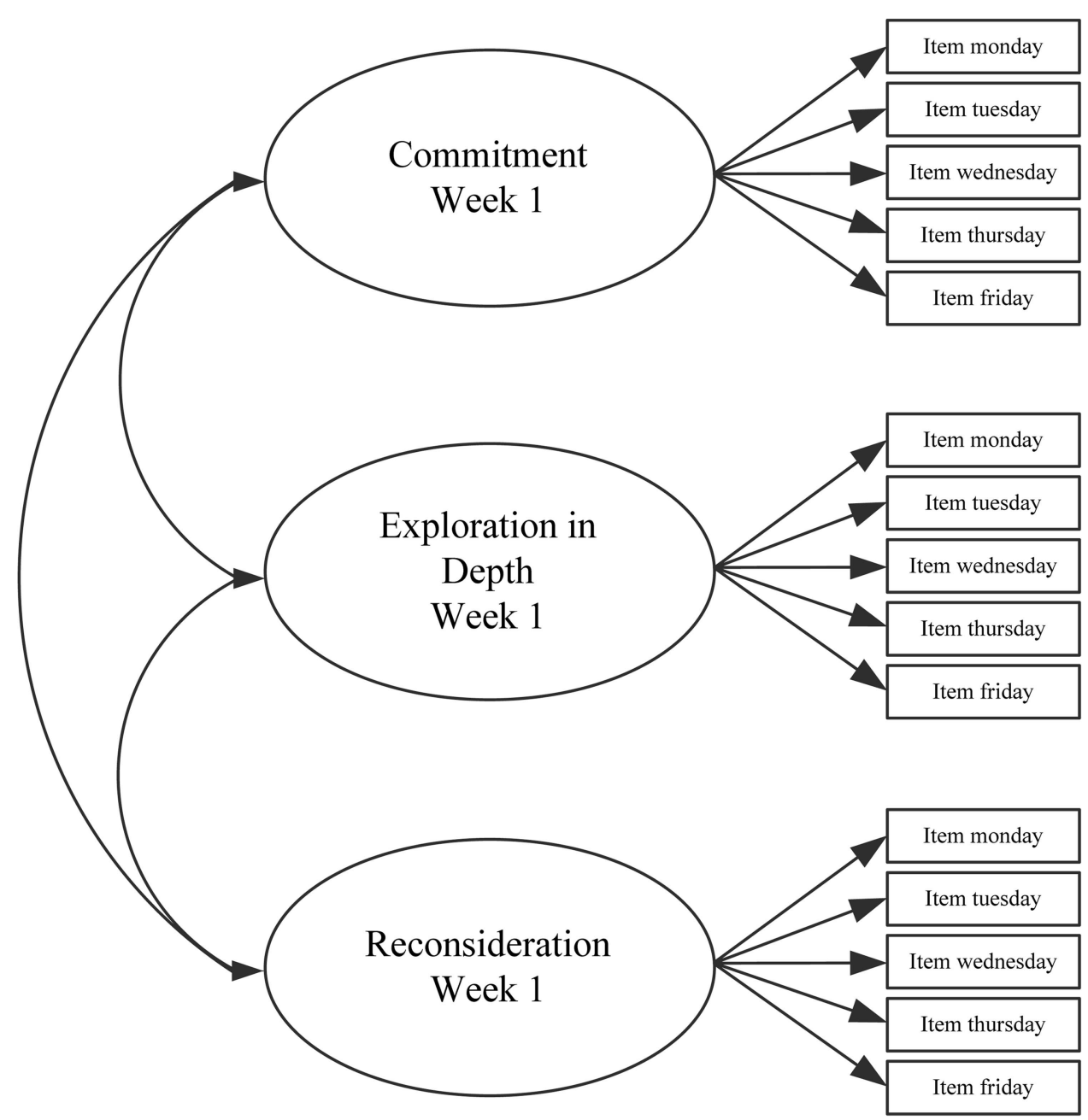

Figure 1. Measurement model for testing measurement invariance of days within a week (here depicted for Week 1). In total, 15 sets of models were tested for Interpersonal Identity and 15 sets of models for Educational Identity.

girls. In each subsequent stage of invariance testing, constraints across sex were added to the multigroup factor model. Again, the five daily indicator items of 1 measurement week were forced to load on the respective factors (i.e., commitment, exploration in depth, and reconsideration during that particular measurement week). This factor structure was the same for every measurement week. Invariance across sex was only assessed for days within weeks and not for weeks within years or for average days across years, because the dayswithin-weeks model was the most fundamental model using the smallest measurement unit (i.e., days) on which the subsequent models were based.

The third set of models tested longitudinal measurement invariance across measurement weeks within years (one set of models per year, resulting in five sets of models across 5 years per identity domain). In this model, the five daily indicator items, all covering 1 day of the week, loaded on the associated factor per week (e.g., Commitment Week 1, Exploration in Depth Week 1, and Reconsideration Week 1). The constraints were set so that the factor structure was the same for all measurement weeks of a particular year (see Figure 3).
In the fourth model set, we tested for longitudinal measurement invariance across years (one model per identity domain). For each year, parcels were computed by taking the mean score of all assessed weekdays within 1 year (e.g., computing the mean score of three Mondays within 1 year). Subsequently, these parcels were used as indicators of the factors per year (see Figure 4). The constraints were set so that the factor structure was the same for each of the 5 years of the study.

The following model specifications were made for purpose of model identification (Vandenberg \& Lance, 2000): (a) The first factor loading for each factor was fixed to 1 for scaling, and (b) the first item's intercept was set to 0 to set the mean of each factor. Factor means, variances, and covariances were freely estimated.

\section{Results}

Because of the large number of models that were analyzed for both interpersonal and educational identity separately, we give 


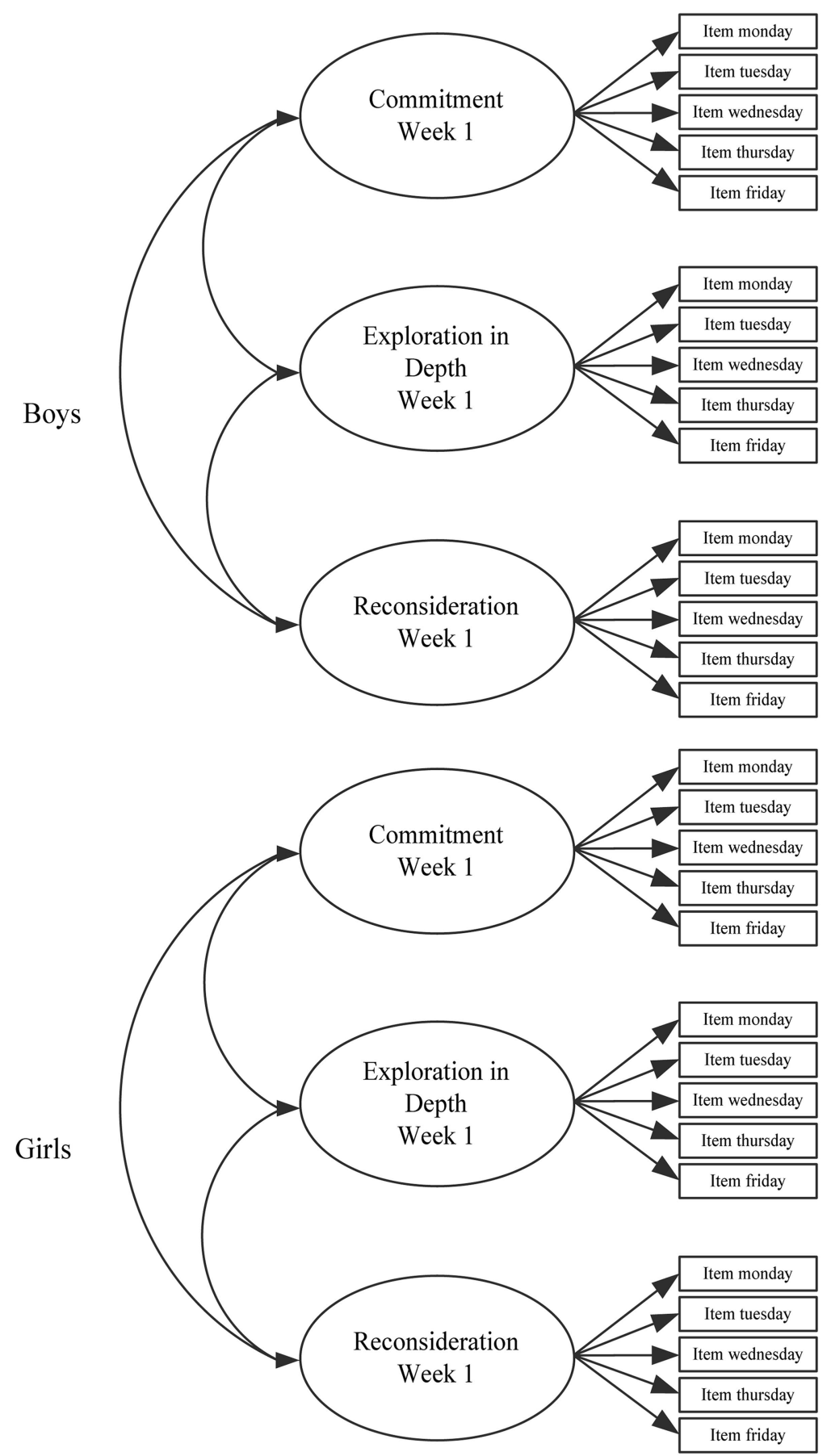

Figure 2. Multigroup measurement model for testing measurement invariance between boys and girls (here depicted for Week 1). In total, 15 sets of models were tested for Interpersonal Identity and 15 sets of models for Educational Identity.

a summary of the fit indices and the model comparisons for the 15 sets of invariance models across days within weeks in Table 1 , for invariance across sex for days within weeks in Table 2, and for measurement invariance of weeks within years in Table 3. The results of each model from these three model sets can be obtained from the authors upon request.

\section{Invariance of Days Within Weeks, 15 Separate Measurement Weeks}

In the first set of models, we tested for measurement invariance of days within weeks (see Figure 1 for the estimated model and Table 1 for the results). First, the configural baseline 


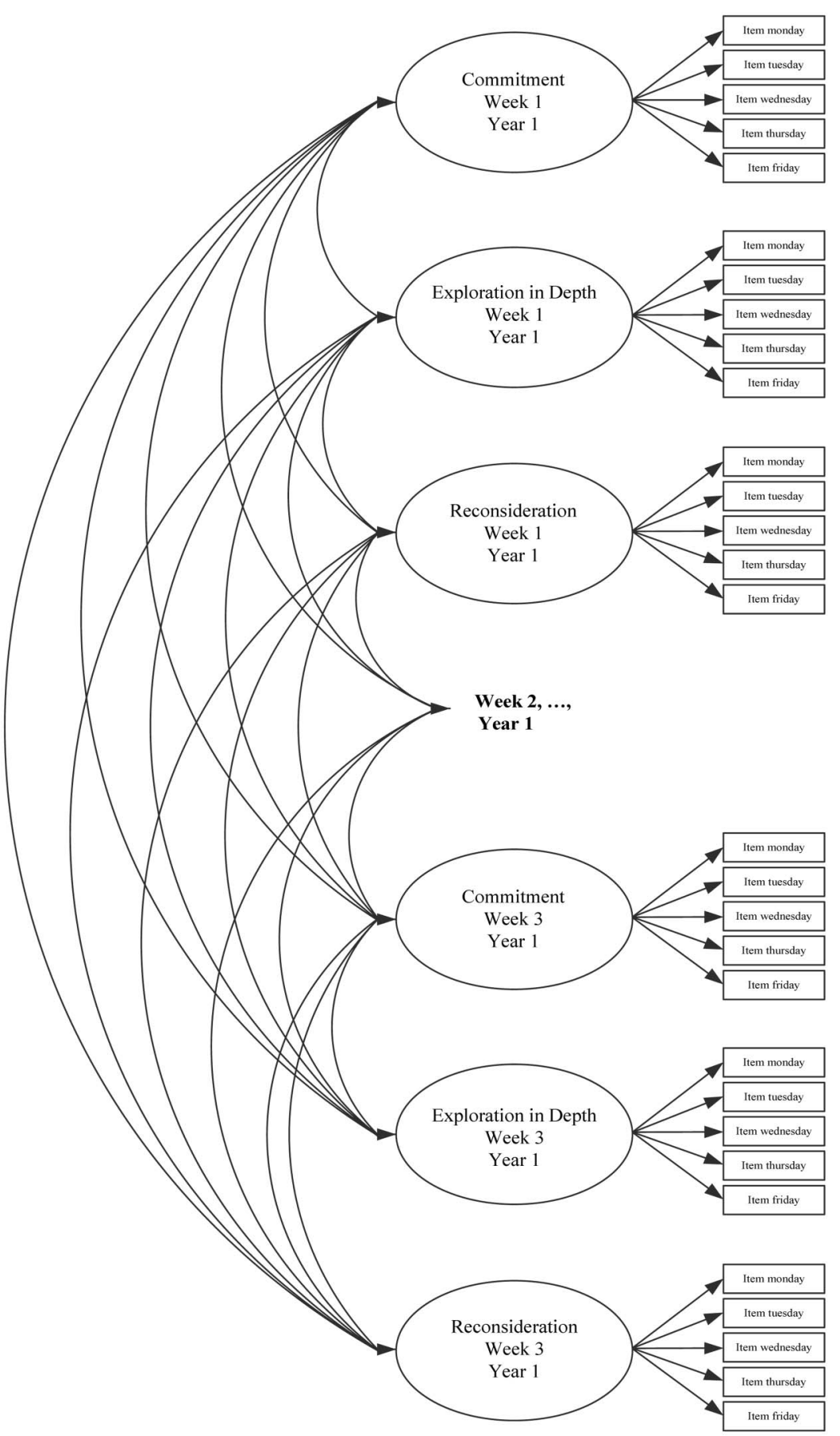

Figure 3. Measurement model for testing measurement invariance of weeks within years (here depicted for Year 1). We tested one set of models for every year over 5 successive years per identity domain (i.e., Interpersonal and Educational).

models indicated that the same three factors and pattern of factor loadings exist across days within weeks. Second, constraining the factor loadings of the same identity dimensions to test for metric invariance did not worsen the model fit. Third, to test for scalar invariance, the intercepts within the same identity dimensions were constrained to be equal across days within weeks, which did not worsen model fit. Finally, to establish strict invariance, residual variances within the same identity dimensions were constrained and results suggested strict invariance across days within weeks. For descriptive purposes only, we report the results of the $\Delta \mathrm{S}-\mathrm{B} \chi^{2}$ difference test as well. Note that, given our large sample, the $\Delta \mathrm{S}-\mathrm{B} \chi^{2}$ test is more conser- 


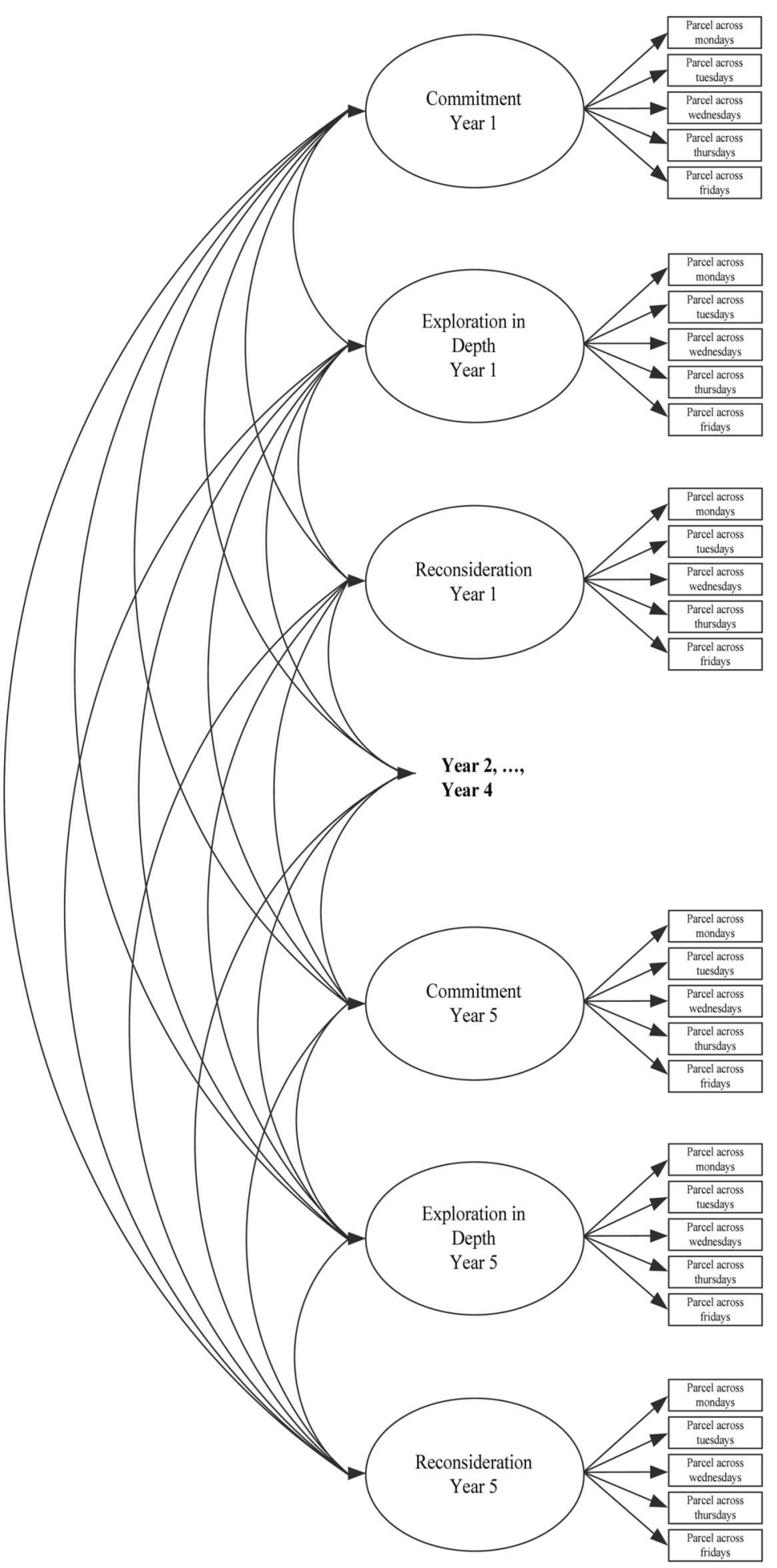

Figure 4. Measurement model for testing measurement invariance across years per identity domain (i.e., Interpersonal and Educational). Note. For each year, parcels were computed by taking the mean score of, for example, the three Mondays within 1 year. 
Table 1

Average Fit Statistics for Measurement Invariance Across Days Within Weeks of Interpersonal and Educational Identity

\begin{tabular}{|c|c|c|c|c|c|c|c|c|c|c|}
\hline \multicolumn{11}{|c|}{ Longitudinal measurement invariance of identity: Days within 15 separate measurement weeks } \\
\hline & $\mathrm{S}-\mathrm{B} \chi^{2}$ & $\Delta \mathrm{S}-\mathrm{B} \chi^{2}$ & $\Delta d f$ & $p$ & CFI & $\Delta \mathrm{CFI}$ & RMSEA & $\triangle$ RMSEA & SRMR & $\overline{\Delta S R M R}$ \\
\hline \multicolumn{11}{|c|}{ Interpersonal Identity (15 models) } \\
\hline Model 1: Configural & 188.59 & & & & 0.96 & & 0.054 & & 0.036 & \\
\hline Model 2: Metric & 201.23 & 12.64 & 9 & .42 & 0.96 & 0.011 & 0.052 & -0.002 & 0.046 & 0.011 \\
\hline Model 3: Scalar & 213.31 & 12.08 & 9 & .41 & 0.96 & 0.001 & 0.051 & -0.002 & 0.048 & 0.001 \\
\hline Model 4: Strict & 231.32 & 18.02 & 12 & .28 & 0.95 & 0.001 & 0.049 & -0.002 & 0.049 & 0.001 \\
\hline \multicolumn{11}{|c|}{ Educational Identity (15 models) } \\
\hline Model 1: Configural & 145.21 & & & & 0.97 & & 0.040 & & 0.038 & \\
\hline Model 2: Metric & 156.56 & 11.65 & 9 & .23 & 0.97 & -0.001 & 0.039 & -0.001 & 0.048 & 0.010 \\
\hline Model 3: Scalar & 175.00 & $18.14^{*}$ & 9 & .03 & 0.96 & -0.005 & 0.040 & 0.001 & 0.051 & 0.003 \\
\hline Model 4: Strict & 199.40 & $24.40^{*}$ & 12 & .01 & 0.96 & -0.006 & 0.040 & 0.000 & 0.056 & 0.005 \\
\hline
\end{tabular}

Note. $\mathrm{CFI}=$ comparative fit index; $\mathrm{CFI}$ values across all models summarized ranged between 0.91 and $0.99 . \Delta \mathrm{CFI}=$ change in $\mathrm{CFI}$; RMSEA $=$ root mean-square error of approximation; $\triangle \mathrm{RMSEA}=$ change in RMSEA; SRMR = standardized root mean-square residual; $\Delta \mathrm{SRMR}=$ change in SRMR; $\mathrm{S}-\mathrm{B} \chi^{2}=$ Satorra-Bentler adjusted $\chi^{2}$ test statistic; $\Delta \mathrm{S}-\mathrm{B} \chi^{2}=$ change in $\mathrm{S}-\mathrm{B} \chi^{2} . p=$ average $p$ value across model comparisons, based on $\mathrm{S}-\mathrm{B} \chi^{2}$ difference testing $\left(\Delta \mathrm{S}-\mathrm{B} \chi^{2}\right)$. Given our large sample, and because $\mathrm{S}-\mathrm{B} \chi^{2}$ is sensitive to sample size, we only used $\mathrm{S}-\mathrm{B} \chi^{2}$ and $\Delta \mathrm{S}$ - $\mathrm{B} \chi^{2}$ for descriptive purposes (Chen, 2007; Cheung \& Rensvold, 2002; Kline, 2010). Average fit indices of the 15 models (i.e., one model per week) per identity domain are reported. All S-B $\chi^{2}$ values were significant at $p<.05$, except for one model with $p>.05 . \Delta \mathrm{S}-\mathrm{B} \chi^{2}$ : Asterisk indicates significant $\chi^{2}$ value based on change in $d f$, where $9=$ critical value $\geq 16.92,12$ critical value $\geq 21.03$. Sixteen of 45 individual $p$ values and 11 out of 45 individual $p$ values were significant for Interpersonal Identity and Educational Identity, respectively.

vative because of its sensitivity to sample size. The $\Delta S-B \chi^{2}$ test was significant for measurement invariance of days within weeks when comparing scalar versus metric invariance and when comparing strict versus scalar measurement invariance for the educational identity domain. However, our models still met the criteria for measurement invariance because change scores on the CFI, RMSEA, and SRMR were below the specified thresholds. The $\Delta \mathrm{S}-\mathrm{B} \chi^{2}$ test did not reach significance when comparing different levels of measurement invariance within the interpersonal identity domain. In sum, both interpersonal and educational identity showed measurement invariance across days within weeks.

\section{Invariance of Days Within Weeks Across Sex, 15 Separate Measurement Weeks}

In the second set of models, we tested for measurement invariance of interpersonal and educational identity of days within weeks across sex (see Figure 2 for the estimated model and Table 2 for the results). All configural models indicated adequate fit. Subsequently, metric invariance was established by constraining the factor loadings of the same identity dimensions to be equal between boys and girls and results indicated that the models were invariant. Constraining the intercepts within dimensions to assess scalar invariance did not result in significant deterioration of

Table 2

Average Fit Statistics for Measurement Invariance Across Sex for Interpersonal and Educational Identity

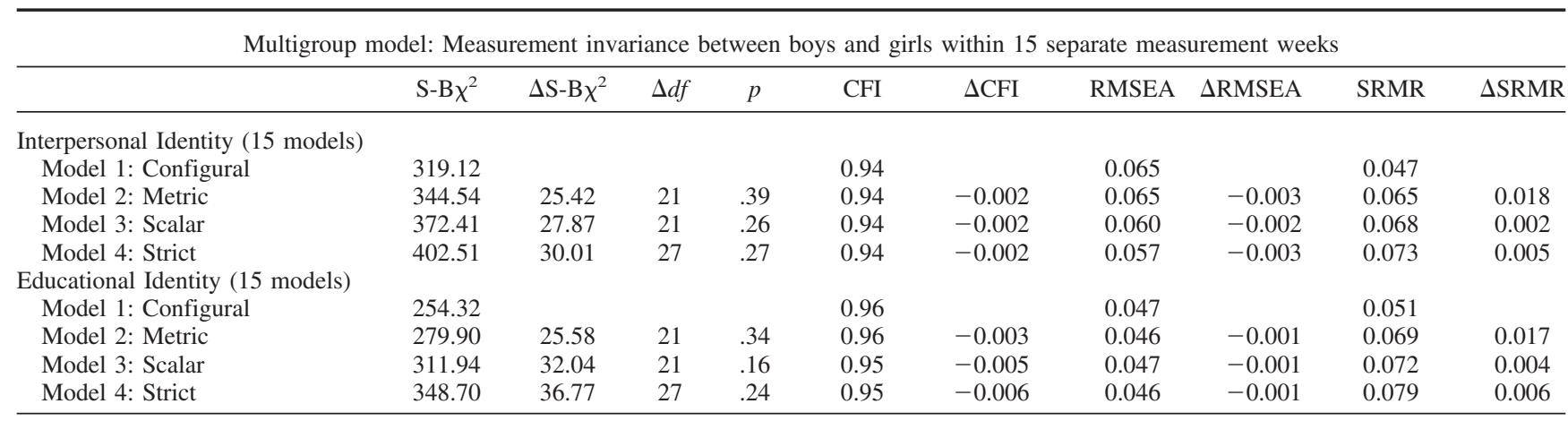

Note. $\mathrm{CFI}=$ comparative fit index; CFI values across all models summarized ranged between 0.91 and $0.98 . \Delta \mathrm{CFI}=$ change in CFI; RMSEA $=$ root mean-square error of approximation; $\triangle \mathrm{RMSEA}=$ change in RMSEA; SRMR = standardized root mean-square residual; $\Delta \mathrm{SRMR}=$ change in SRMR; $\mathrm{S}-\mathrm{B} \chi^{2}=$ Satorra-Bentler adjusted $\chi^{2}$ test statistic; $\Delta \mathrm{S}-\mathrm{B} \chi^{2}=$ change in Satorra-Bentler adjusted $\chi^{2}$ test statistic; $p=$ average $p$ value across model comparisons, based on $\mathrm{S}-\mathrm{B} \chi^{2}$ difference testing $\left(\Delta \mathrm{S}-\mathrm{B} \chi^{2}\right)$. Average fit indices of the 15 models (i.e., one model per week) per identity domain are reported All S-B $\chi^{2}$ values were significant at $p<.001 . \Delta \mathrm{S}-\mathrm{B} \chi^{2}$ : Asterisk indicates significant $\chi^{2}$ value based on change in $d f$, where $21=$ critical value $\geq 32.67$, $27=$ critical value $\geq 40.11$ ). Thirteen of 45 individual $p$ values and 12 of 45 individual $p$ values were significant for Interpersonal Identity and Educational Identity, respectively. 
Table 3

Average Fit Statistics for Measurement Invariance of Interpersonal and Educational Identity Across Measurement Weeks Within 5 Separate Years

\begin{tabular}{|c|c|c|c|c|c|c|c|c|c|c|}
\hline \multicolumn{11}{|c|}{ Longitudinal measurement invariance of identity: Weeks within 5 separate years } \\
\hline & $\mathrm{S}-\mathrm{B} \chi^{2}$ & $\Delta \mathrm{S}-\mathrm{B} \chi^{2}$ & $\Delta d f$ & $p$ & $\mathrm{CFI}$ & $\Delta \mathrm{CFI}$ & RMSEA & $\Delta$ RMSEA & SRMR & $\overline{\Delta \text { SRMR }}$ \\
\hline \multicolumn{11}{|c|}{ Interpersonal Identity (5 models) } \\
\hline Model 1: Configural & 1429.71 & & & & 0.95 & & 0.037 & & 0.040 & \\
\hline Model 2: Metric & 1473.22 & 43.51 & 33 & .35 & 0.95 & -0.001 & 0.037 & 0.000 & 0.045 & 0.005 \\
\hline Model 3: Scalar & 1512.44 & 39.22 & 33 & .33 & 0.95 & -0.001 & 0.036 & 0.000 & 0.045 & 0.000 \\
\hline Model 4: Strict & 1578.86 & $66.43^{*}$ & 42 & .14 & 0.94 & -0.002 & 0.036 & 0.000 & 0.047 & 0.001 \\
\hline \multicolumn{11}{|c|}{ Educational Identity (5 models) } \\
\hline Model 1: Configural & 1262.58 & & & & 0.95 & & 0.030 & & 0.044 & \\
\hline Model 2: Metric & 1305.28 & 42.70 & 33 & .27 & 0.95 & -0.001 & 0.030 & 0.000 & 0.049 & 0.005 \\
\hline Model 3: Scalar & 1377.24 & $71.96^{*}$ & 33 & .01 & 0.95 & -0.005 & 0.031 & 0.001 & 0.049 & 0.001 \\
\hline Model 4: Strict & 1464.57 & $87.33^{*}$ & 42 & .19 & 0.94 & -0.005 & 0.032 & 0.000 & 0.053 & 0.004 \\
\hline
\end{tabular}

Note. $\quad \mathrm{CFI}=$ comparative fit index; CFI values across all models summarized ranged between 0.91 and $0.96 . \Delta \mathrm{CFI}=$ change in CFI; RMSEA $=$ root-mean-square error of approximation; $\triangle \mathrm{RMSEA}=$ change in RMSEA; SRMR = standardized root-mean-square residual; $\triangle \mathrm{SRMR}=\mathrm{change}$ in SRMR. $\mathrm{S}-\mathrm{B} \chi^{2}=$ Satorra-Bentler adjusted chi-square test statistic; $\Delta \mathrm{S}-\mathrm{B} \chi^{2}=$ change in $\mathrm{S}-\mathrm{B} \chi^{2} ; p=$ average $p$ value across model comparisons, based on $\mathrm{S}-\mathrm{B} \chi^{2}$ difference testing $\left(\mathrm{S}-\mathrm{B} \chi^{2}\right)$. Average fit indices of the five models (i.e., one model per year) per identity domain are reported. All $\mathrm{S}-\mathrm{B} \chi^{2}$ values were significant at $p<.001 . \Delta \mathrm{S}-\mathrm{B} \chi^{2}$ : asterisk indicates significant $\chi^{2}$ value based on change in $d f$, where $33=$ critical value $\geq 47.40,42=$ critical value $\geq$ 58.12. Seven of 15 individual $p$ values and 6 of 15 individual $p$ values were significant for Interpersonal Identity and Educational Identity, respectively.

model fit. Finally, constraining the residual variances for the indicators of the same identity dimensions to be equal across sex showed the models to be invariant across boys and girls, thereby suggesting strict measurement invariance. In line with our finding of measurement invariance across days within weeks between boys and girls, based on the change scores on the CFI, RMSEA and SRMR, the $\Delta \mathrm{S}-\mathrm{B} \chi^{2}$ test was not significant at any stage of measurement invariance for both the interpersonal and educational identity domains. Thus, both interpersonal and educational identity showed measurement invariance for boys and girls.

\section{Invariance Across Measurement Weeks Within 5 Separate Years}

In the third set of models, we examined whether interpersonal and educational identity showed measurement invariance across measurement weeks within years (see Figure 3 and Table 3 ). The configural model indicated adequate fit with the data. Subsequently constraining the factor loadings and intercepts within identity dimensions to be equal across weeks within years showed adequate fit, suggesting metric and scalar invariance, respectively. Finally, constraining the residual variances within identity dimensions to be equal across weeks within years did not result in a poorer fitting model, implying strict invariance across weeks within years. The $\Delta \mathrm{S}-\mathrm{B} \chi^{2}$ test was significant for measurement invariance of weeks within years when comparing strict versus scalar measurement invariance within the interpersonal identity domain. With regard to the educational identity domain, the $\Delta S$ $\mathrm{B} \chi^{2}$ test was significant when comparing scalar versus metric, and when comparing strict versus scalar measurement invariance. However, our models still met the criteria for measurement invariance because change scores on the CFI, RMSEA, and SRMR were all below the specified thresholds. Hence, both the models concerning interpersonal and educational identity revealed measurement invariance across measurement weeks within separate assessment years.

\section{Invariance Across Years}

In the final set of models, using parcels, we tested for measurement invariance across 5 years by taking the mean score of every day within 1 year (e.g., the mean of three Mondays within 1 year; see Figure 4 and Table 4). The configural model, in which we tested for an equal factor structure across the 5 years, had a good fit with the data. Next, testing the models for metric, scalar, and strict invariance all showed good fit statistics and no significant worsening of the model fits. Regarding the $\Delta S-B \chi^{2}$ test of invariance across years, comparison of the strict versus the scalar measurement-invariance model reached significance within the interpersonal identity domain. Concerning the educational identity domain, the $\Delta \mathrm{S}-\mathrm{B} \chi^{2}$ test was significant when comparing the scalar versus the metric measurement-invariance model, and when comparing the strict versus the scalar measurement-invariance model. However, our models still met the criteria for measurement invariance because change scores on the CFI, RMSEA, and SRMR were all below the specified thresholds.

Therefore, measurement invariance was supported for interpersonal and educational identity across years.

\section{Discussion}

In this study, we examined whether adolescents' daily identity reports exhibited measurement invariance across time and sex. Results indicate that daily identity reports showed consistent configural, metric, scalar, and strict measurement invariance across days within weeks, across days within weeks for boys and girls, across weeks within years, and across 5 years. Measurement invariance was found for two important developmental domains of identity, namely interpersonal and educational identity. We used data from 497 adolescents who were followed from age 13 to age 18 and reported on interpersonal and educational domains of identity. To our best knowledge, this is the first study looking at measurement invariance of daily diary reports on identity across adolescence. 
Table 4

Fit Statistics for Measurement Invariance of Weekday Averages Regarding Interpersonal and Educational Identity Across Years

\begin{tabular}{|c|c|c|c|c|c|c|c|c|c|c|}
\hline \multicolumn{11}{|c|}{ Longitudinal measurement invariance of identity: Across years } \\
\hline & $\mathrm{S}-\mathrm{B} \chi^{2}$ & $\Delta \mathrm{S}-\mathrm{B} \chi^{2}$ & $\Delta d f$ & $p$ & CFI & $\Delta \mathrm{CFI}$ & RMSEA & $\Delta$ RMSEA & SRMR & $\Delta$ SRMR \\
\hline \multicolumn{11}{|l|}{ Interpersonal Identity } \\
\hline Model 1: Configural & 4176.26 & & & & 0.94 & & 0.036 & & 0.031 & \\
\hline Model 2: Metric & 4246.98 & 70.72 & 57 & .10 & 0.94 & -0.001 & 0.035 & -0.001 & 0.034 & 0.003 \\
\hline Model 3: Scalar & 4309.36 & 62.38 & 57 & .45 & 0.94 & -0.000 & 0.035 & 0.000 & 0.035 & 0.001 \\
\hline Model 4: Strict & 4676.15 & $366.79^{*}$ & 72 & .00 & 0.93 & -0.010 & 0.038 & 0.003 & 0.039 & 0.004 \\
\hline \multicolumn{11}{|l|}{ Educational Identity } \\
\hline Model 1: Configural & 3502.71 & & & & 0.96 & & 0.027 & & 0.036 & \\
\hline Model 2: Metric & 3570.71 & 68.00 & 57 & .15 & 0.96 & -0.000 & 0.027 & 0.000 & 0.040 & 0.004 \\
\hline Model 3: Scalar & 3729.70 & $159.00^{*}$ & 57 & .00 & 0.95 & -0.005 & 0.028 & 0.001 & 0.040 & 0.000 \\
\hline Model 4: Strict & 3983.48 & $253.30^{*}$ & 72 & .00 & 0.94 & -0.009 & 0.030 & 0.002 & 0.044 & 0.004 \\
\hline
\end{tabular}

Note. $\mathrm{S}-\mathrm{B} \chi^{2}=$ Satorra-Bentler adjusted $\chi^{2}$ test statistic; $\Delta \mathrm{S}-\mathrm{B} \chi^{2}=$ change in $\mathrm{S}-\mathrm{B} \chi^{2} ; \mathrm{CFI}=$ comparative fit index; $\Delta \mathrm{CFI}=$ change in $\mathrm{CFI} ; \mathrm{RMSEA}=$ root mean-square error of approximation; $\triangle$ RMSEA = change in RMSEA; SRMR $=$ standardized rootmean-square residual; $\Delta$ SRMR $=$ change in SRMR . All S-B $\chi^{2}$ values were significant at $p<.001 . \Delta \mathrm{S}-\mathrm{B} \chi^{2}$ : Asterisk indicates significant $\chi^{2}$ value based on change in $d f$, where $57=$ critical value $\geq 75.62$, $72=$ critical value $\geq 92.81$

The fact that daily diary reports showed metric invariance (i.e., comparable factor loadings) implies that daily reports, for example, within weeks, are equally important indicators of the shortterm assessed underlying latent identity factor (i.e., in our study, assessed per week), both across time and sex. Because metric invariance was established, it is possible to compare relationships between identity and other outcomes across time and sex. In addition, our finding of scalar invariance implies that total scores can be used for mean comparisons of identity scores across different time points and between boys and girls. Therefore, differences in mean scores across time and sex can be attributed to differences in the underlying latent identity factor rather than measurement artifacts (Little, 2013). Finally, we found evidence for strict measurement invariance, which suggests that these identity constructs are assessed with the same amount of random error across different time points and between boys and girls (van de Schoot et al., 2012).

Our finding that daily diary methods show measurement invariance over time has implications for the assessment of identity in future studies. For example, daily scores within a week can be aggregated into a more robust score, such as a weekly mean-level identity score or a variability score (Shiffman, Stone, \& Hufford, 2008). These aggregated scores may be a useful level at which to study development of identity processes across adolescence that can inform both shorter and larger time interval assessments of identity. Indeed, research on conflict frequency in adolescent's close relationships shows that the use of daily diary methods provided the most reliable assessment strategy (Burk, Denissen, Van Doorn, Branje, \& Laursen, 2009). It has been suggested that the use of daily diary methods is reliable due to fewer recall biases when answering a question (Shiffman et al., 2008).

Our results open venues for future research. Because we found evidence for scalar measurement invariance across days within weeks, it is possible to study structural (in)variance such as shortterm mean-level development of identity or short-term mean-level differences in identity development between boys and girls. For example, because MAMA-cycles (Stephen et al., 1992) are expected to occur shortly before and around life events, daily diary reports can be used to investigate these identity-formation pro- cesses in more detail. In addition to using daily assessments of identity to measure short-term development, the current study showed that these short-term assessments can be used across 5 years (i.e., between 13 and 18 years of age). Therefore, daily diary reports may be used to make meaningful comparisons on identity development both across shorter and longer time intervals.

Besides studying short-term mean development, daily reports on adolescents identity can be used to create, for example, aggregated weekly variability scores. As suggested by Campbell (1990), future researchers using daily diary reports may empirically investigate developmental principles as to whether mean levels or fluctuations in identity-formation processes are better predictors of adolescent's outcomes.

The current study showed that daily diary reports of identity can be used to measure the same construct across time and sex. However, another important quality of a measurement instrument is its sensitivity to change (e.g., Vermeersch, Lambert, \& Burlingame, 2000), which is the ability of an instrument to measure true change over time. Although we did not investigate this in our study, previous research using the same sample showed withinperson change of identity on a day-to-day basis across adolescence (Klimstra et al., 2015). Moreover, this change in identity from one day to another is related to change in adjustment outcomes. For example, a longitudinal study on the relation between daily identity and daily mood showed that lower levels of identity commitments were related to higher levels of anger and sadness (Klimstra et al., 2015). Similarly, day-to-day fluctuations in adolescents' identity predicted later anxiety and depression (Schwartz et al., 2011).

With regard to clinical relevance, daily diary assessments of identity may be used to investigate whether day-to-day variability in identity processes predict, for example, symptoms of borderline personality disorder. For instance, using experience-sampling methods, it has been found that undergraduate students with borderline personality features were characterized by more day-to-day fluctuations in self-esteem, especially in reaction to daily interpersonal stressors, such as feelings of rejection (Zeigler-Hill \& Abraham, 2006). 


\section{Strengths, Limitations, and Future Directions}

The present study is characterized by several strengths. The most important strength is its extensive longitudinal design that included daily diary reports on identity covering the whole period of adolescence across 5 years. Second, we investigated measurement invariance of two important developmental identity domains in adolescence: Interpersonal Identity and Educational Identity (Grotevant, 1987). Third, the present study was the first to investigate measurement invariance of a short-term assessment of identity across time and sex. Therefore, we were able to establish in this study that daily diary assessments can be used to compare identity-formation processes across adolescence at different time intervals, ranging from weeks to years.

In addition to these strengths, some limitations of the study need to be mentioned. First, the sample consisted of primarily middleclass Dutch adolescents from relatively high SES families, which imposes questions as to whether our findings can be generalized to lower SES samples. Second, the focus of the present study was limited to the assessment of measurement invariance across time and sex. Although previous research on adolescent conflict in close relationships has shown that daily diary assessments have many advantages over assessments with larger time intervals (Burk et al., 2009), research on identity is less clear. Future studies are needed to examine, for example, whether these daily diary assessments of identity show incremental validity in predicting adolescents' adjustment outcomes compared with larger time interval assessments. Third, as we assessed each identity dimension with a single-item question, we were not able to examine measurement invariance for daily latent constructs of identity. Fourth, despite our use of multiple indicators for the assessment of measurement invariance, such as the change scores in CFI, RMSEA, and SRMR, there is not yet much evidence for the best fit indices that can be used to evaluate longitudinal measurement invariance. To our knowledge, only the CFI has been validated in simulation studies (Chen, 2007), but not yet for the assessment of longitudinal measurement invariance. Moreover, $\chi^{2}$ difference tests for model comparison are not considered appropriate because of their sensitivity to sample size (e.g., Kline, 2010). Future simulation studies are needed to validate the use of different fit indices to evaluate longitudinal measurement invariance.

Despite these limitations, this is the first study to test whether daily diary assessments of identity are measurement-invariant across time and sex. Our results indicate that daily diary reports can be used to further increase our knowledge about developmental processes of identity formation during adolescence, both across shorter and longer time intervals and across boys and girls.

\section{References}

American Psychiatric Association, DSM-5 Task Force. (2013). DSM-5: Diagnostic and statistical manual of mental disorders (5th ed.). Arlington, VA: American Psychiatric Publishing.

Bollen, K. (1989). Structural equations with latent variables. New York, NY: Wiley. http://dx.doi.org/10.1002/9781118619179

Burk, W. J., Denissen, J., Van Doorn, M. D., Branje, S. J., \& Laursen, B. (2009). The vicissitudes of conflict measurement: Stability and reliability in the frequency of disagreements. European Psychologist, 14, 153159. http://dx.doi.org/10.1027/1016-9040.14.2.153
Byrne, B. M. (2012). Structural equation modeling with Mplus: Basic concepts, applications, and programming. New York, NY: Routledge.

Campbell, J. D. (1990). Self-esteem and clarity of the self-concept. Journal of Personality and Social Psychology, 59, 538-549. http://dx.doi.org/ 10.1037/0022-3514.59.3.538

Chen, F. F. (2007). Sensitivity of goodness of fit indexes to lack of measurement invariance. Structural Equation Modeling, 14, 464-504. http://dx.doi.org/10.1080/10705510701301834

Cheung, G. W., \& Rensvold, R. B. (2002). Evaluating goodness-of-fit indexes for testing measurement invariance. Structural Equation Modeling, 9, 233-255. http://dx.doi.org/10.1207/S15328007SEM0902_5

Crocetti, E., Rubini, M., \& Meeus, W. (2008). Capturing the dynamics of identity formation in various ethnic groups: Development and validation of a three-dimensional model. Journal of Adolescence, 31, 207-222. http://dx.doi.org/10.1016/j.adolescence.2007.09.002

Erikson, E. (1968). Identity, youth and crisis. New York, NY: Norton.

Feenstra, D. J., Hutsebaut, J., Verheul, R., \& van Limbeek, J. (2014). Identity: Empirical contribution. Changes in the identity integration of adolescents in treatment for personality disorders. Journal of Personality Disorders, 28, 101-112. http://dx.doi.org/10.1521/pedi.2014.28.1.101

Graber, J. A., Brooks-Gunn, J. E., \& Petersen, A. C. (1996). Transitions through adolescence: Interpersonal domains and context. Hillsdale, $\mathrm{NJ}$ Erlbaum.

Grotevant, H. (1987). Toward a process model of identity formation. Journal of Adolescent Research, 2, 203-222. http://dx.doi.org/10.1177/ 074355488723003

Heise, D. R. (1969). Separating reliability and stability in test-retest correlation. American Sociological Review, 34, 93-101. http://dx.doi .org/10.2307/2092790

Klimstra, T. A., Kuppens, P., Luyckx, K., Branje, S., Hale, W. W., III, Oosterwegel, A., . . Meeus, W. H. (2015). Daily dynamics of adolescent mood and identity. Journal of Research on Adolescence [Advance online publication]. http://dx.doi.org/10.1111/jora.12205

Klimstra, T. A., Luyckx, K., Hale, W. A., III, Frijns, T., van Lier, P. A., \& Meeus, W. H. (2010). Short-term fluctuations in identity: Introducing a micro-level approach to identity formation. Journal of Personality and Social Psychology, 99, 191-202. http://dx.doi.org/10.1037/a0019584

Kline, R. B. (2010). Principles and practice of structural equation modeling (3rd ed.). New York, NY: Guilford Press.

Kroger, J., Martinussen, M., \& Marcia, J. E. (2010). Identity status change during adolescence and young adulthood: A meta-analysis. Journal of Adolescence, 33, 683-698. http://dx.doi.org/10.1016/j.adolescence.2009 .11 .002

Lichtwarck-Aschoff, A., van Geert, P., Bosma, H., \& Kunnen, S. (2008). Time and identity: A framework for research and theory formation. Developmental Review, 28, 370-400. http://dx.doi.org/10.1016/j.dr 2008.04.001

Little, T. D. (2013). Longitudinal structural equation modeling. New York, NY: Guilford Press.

Luyckx, K., Goossens, L., \& Soenens, B. (2006). A developmental contextual perspective on identity construction in emerging adulthood: Change dynamics in commitment formation and commitment evaluation. Developmental Psychology, 42, 366-380. http://dx.doi.org/ 10.1037/0012-1649.42.2.366

Meeus, W., van de Schoot, R., Keijsers, L., Schwartz, S. J., \& Branje, S. (2010). On the progression and stability of adolescent identity formation: A five-wave longitudinal study in early-to-middle and middle-to-late adolescence. Child Development, 81, 1565-1581. http://dx.doi.org/ 10.1111/j.1467-8624.2010.01492.x

Muthén, L. K., \& Muthén, B. O. (1998-2012). Mplus user's guide (7th ed.). Los Angeles, CA: Authors.

Nesselroade, J. R., \& Molenaar, P. C. M. (2010). Emphasizing intraindividual variability in the study of development over the life span: Con- 
cepts and issues. In R. M. Lerner \& W. F. Overton (Eds.), The handbook of lifespan development: Vol. 1. Cognition, biology, and methods (pp. 30-54). Hoboken, NJ: Wiley. http://dx.doi.org/10.1002/9780470880166 .hlsd001002

Schwartz, S. J., Klimstra, T. A., Luyckx, K., Hale, W. W., III, Frijns, T., Oosterwegel, A., . . Meeus, W. H. (2011). Daily dynamics of personal identity and self-concept clarity. European Journal of Personality, 25, 373-385. http://dx.doi.org/10.1002/per.798

Shiffman, S., Stone, A. A., \& Hufford, M. R. (2008). Ecological momentary assessment. Annual Review of Clinical Psychology, 4, 1-32. http:// dx.doi.org/10.1146/annurev.clinpsy.3.022806.091415

Stephen, J., Fraser, E., \& Marcia, J. E. (1992). Moratorium-achievement (MAMA) cycles in lifespan identity development: Value orientations and reasoning system correlates. Journal of Adolescence, 15, 283-300. http://dx.doi.org/10.1016/0140-1971(92)90031-Y

Vandenberg, R. J., \& Lance, C. E. (2000). A review and synthesis of the measurement invariance literature: Suggestions, practices, and recommendations for organizational research. Organizational Research Methods, 3, 4-70. http://dx.doi.org/10.1177/109442810031002

van de Schoot, R., Lugtig, P., \& Hox, J. (2012). A checklist for testing measurement invariance. European Journal of Developmental Psychology, 9, 486-492. http://dx.doi.org/10.1080/17405629.2012.686740
Vater, A., Schröder-Abé, M., Weißgerber, S., Roepke, S., \& Schütz, A. (2015). Self-concept structure and borderline personality disorder: Evidence for negative compartmentalization. Journal of Behavior Therapy and Experimental Psychiatry, 46, 50-58. http://dx.doi.org/10.1016/j .jbtep.2014.08.003

Vermeersch, D. A., Lambert, M. J., \& Burlingame, G. M. (2000). Outcome Questionnaire: Item sensitivity to change. Journal of Personality Assessment, 74, 242-261. http://dx.doi.org/10.1207/S15327752JPA7402_6

Wilkinson-Ryan, T., \& Westen, D. (2000). Identity disturbance in borderline personality disorder: An empirical investigation. The American Journal of Psychiatry, 157, 528-541. http://dx.doi.org/10.1176/appi.ajp 157.4.528

Yip, T., \& Fuligni, A. J. (2002). Daily variation in ethnic identity, ethnic behaviors, and psychological well-being among American adolescents of Chinese descent. Child Development, 73, 1557-1572. http://dx.doi .org/10.1111/1467-8624.00490

Zeigler-Hill, V., \& Abraham, J. (2006). Borderline personality features: Instability of self-esteem and affect. Journal of Social and Clinical Psychology, 25, 668-687. http://dx.doi.org/10.1521/jscp.2006.25.6.668

Received January 23, 2015

Revision received June 12, 2015

Accepted June 15, 2015

\section{Correction to Becht et al. (2015)}

In the article "Assessment of Identity During Adolescence Using Daily Diary Methods: Measurement Invariance Across Time and Sex" by Andrik I. Becht, Susan J. T. Branje, Wilma A. M. Vollebergh, Dominique F. Maciejewski, Pol A. C. van Lier, Hans M. Koot, Jaap J. A. Denissen, and Wim H. J. Meeus (Psychological Assessment, Advanced online publication. August 10, 2015. http://dx.doi.org/10.1037/pas0000204), the participants should have been reported as $N=494$. No differences were found in the results upon reanalyzing the data with the correct number of participants.

Additionally, the last sentence of the first full paragraph in the Invariance Across Boys and Girls subsection of the Method section should read "In the fourth model, strict invariance was examined, in which the residual variances were constrained to be equal for boys and girls." 